\title{
Neurocomputing Techniques to Predict the 2D Structures by Using Lattice Dynamics of Surfaces
}

\author{
A. Belayadi ${ }^{a, b, *}$, B. Bourahla ${ }^{c}$ And F. Mekideche-Chafa ${ }^{a}$ \\ ${ }^{a}$ University of Science and Technology H. Boumedienne, Department of Physics, Algiers, Algeria \\ ${ }^{b}$ University of M. Bougara, Department of Coating Material and Environmental, Boumerdes, Algeria \\ ${ }^{c}$ Laboratory of Physics and Quantum Chemistry, M. Mammeri University, Tizi-Ouzou, Algeria
}

(Received October 8, 2016)

\begin{abstract}
A theoretical study of artificial neural network modelling, based on vibrational dynamic data for 2D lattice, is proposed in this paper. The main purpose is to establish a neurocomputing model able to predict the $2 \mathrm{D}$ structures of crystal surfaces. In material surfaces, atoms can be arranged in different possibilities, defining several $2 \mathrm{D}$ configurations, such as triangular, square lattices, etc. To describe these structures, we usually employ the Wood notations, which are considered as the simplest manner and the most frequently used to spot the surfaces in physics. Our contribution consists to use the vibration lattice of perfect $2 \mathrm{D}$ structures along with the matrix and Wood notations to build up an input-output set to feed the neural model. The input data are given by the frequency modes over high symmetry points and the group velocity. The output data are given by the basis vectors corresponding to surface reconstruction and the rotation angle which aligns the unit cell of the reconstructed surface. Results showed that the method of collecting the dataset was very suitable for building a neurocomputing model that is able to predict and classify the 2D surface of the crystals. Moreover, the model was able to generate the lattice spacing for a given structure.
\end{abstract}

DOI: 10.12693/APhysPolA.132.1314

PACS/topics: neurocomputing, surface and Wood notation, vibration and lattice dynamics

\section{Introduction}

Neural networks have been widely used for all kinds of applications, such as prediction and classification [1-4], where they have gained their reputation and proved that they can be reliable alternatives to other classification methods. The modelling of physical phenomena is still attracting a significant interest for many issues and has been treated by neural network techniques. For instance, we can quote its applications in nanotechnology [5], electrical conducted solids [6], electromagnetic theory [7], fuel ignition model in combustion theory [8], magnetohydrodynamics (MHD) [9], fluid dynamics problems [10], Troesch's problem arising in plasma physics [11].

The atoms, in solid crystals, are placed in an ordered way; their specific arrangements are conferred by the interatomic forces. These forces play the same roles as the springs in a spring-mass system. Therefore, if an atom has slightly moved from its initial position, it will oscillate around its equilibrium position. As the latter is linked to the other atoms by electrostatic forces, the other atoms of the crystal will also vibrate. Consequently, a vibrational wave will propagate as a deformation within the crystal. These vibrations have a direct impact on the properties of solids [12], like melting temperature, thermal conductivity, frequency modes, velocity, etc. As well as indirect effects appear, in particular, on the resistivity and the attractive interaction that leads to superconductivity [13].

*corresponding author; e-mail: bourahla_boualem@yahoo.fr
In addition, many techniques have been applied to evaluate the elastic and mechanical properties of $2 \mathrm{D}$ system [14], in particular, the Brillouin light scattering from surface acoustic waves [15] and surface acoustic wave spectroscopy [16].

The main interest, in this contribution, is to study the framework of artificial neural network computing. We begin by developing a theoretical approach of a NCM for predicting the 2D lattice of crystal surfaces. The calculations of frequency modes, group velocity, and vector elements corresponding to the matrix and Wood presentation are used to feed in data to the proposed NCM.

This manuscript is organized as follows: in the next section, we describe, briefly, some basic notions of lattice dynamics in crystals. Then, we make use of the vibration properties of perfect 2D lattice to extract the physical parameters that will be used to collect the input-output set of neural model. In Sect. 3, we provide a detailed explanation about data collection as well as the neurocomputing methodology in predicting the 2D structures. In the last section, summary and conclusion of work are provided.

\section{Dynamic of the diatomic perfect 2D structure}

The model system studied, in this work, is schematized in Fig. 1. The representation consists of a diatomic plane containing two different types of atoms.

Under the action of thermal agitation or magnetic radiations, atoms do not keep on a fixed site, but vibrate around their equilibrium positions. The amplitude of displacements depends strongly on the intensity of excitation. 
Assuming that the amplitude of vibration of each atom is small compared to the distance between nearest neighbours, the forces that bind atomic sites will be presented in the harmonic approximation [17].

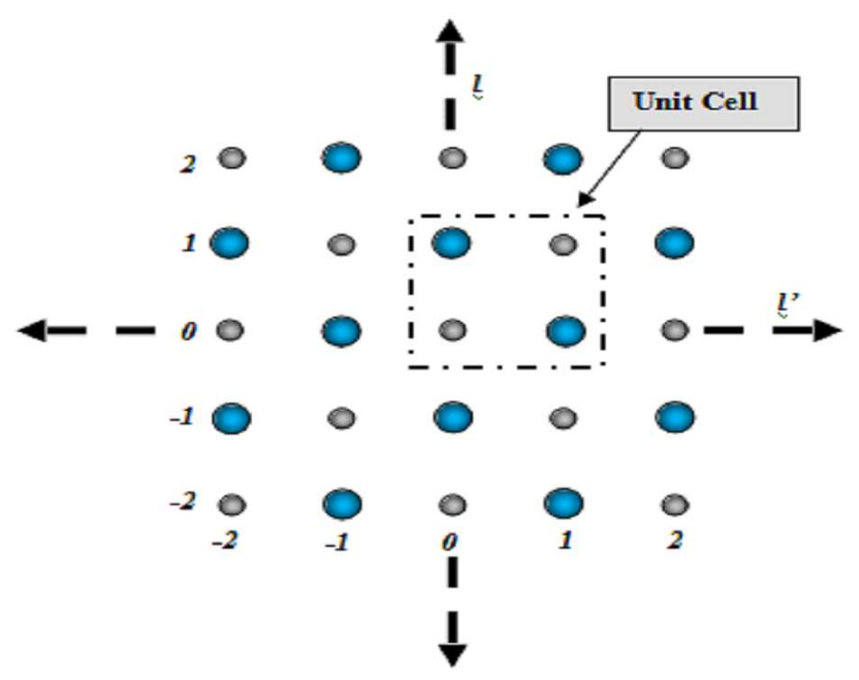

Fig. 1. A schematic representation of infinite diatomic 2 D lattice. In this case, $\ell \equiv(0,1)$ and $\ell^{\prime} \equiv(0,1)$.

Moreover, an infinite 2D crystal, which is characterized by a periodic repetition in the Bloch space, translational symmetry, and periodic boundary conditions, reduces the atomic displacement field. All these assumptions allow us to describe the motion equation of the sites $\ell$ and $\ell^{\prime}$ as

$$
\begin{aligned}
& \omega^{2} m_{\ell} \boldsymbol{u}_{\alpha}(\ell, t)=\sum_{\ell^{\prime} \neq \ell} \sum_{\beta}\left(\frac{r_{\alpha} r_{\beta}}{r^{2}}\right) k\left(\ell, \ell^{\prime}\right)\left(\boldsymbol{u}_{\beta}\left(\ell^{\prime}, t\right)\right. \\
& \left.-\boldsymbol{u}_{\beta}(\ell, t)\right) .
\end{aligned}
$$

The indices $\alpha$ and $\beta$ represent Cartesian co-ordinates, $m_{\ell}$ is the atomic mass for site $\ell$, and $\boldsymbol{u}_{\alpha}(\ell, t)$ is its corresponding displacement vector vibration, $r_{\alpha}$ is the $\alpha$ component of the radius vector, $r$ the distance between $\ell$ and $\ell^{\prime}$ sites, and $k\left(\ell, \ell^{\prime}\right)$ is the elastic constant between the two neighbouring sites.

In the perfect lattice, the previous linear equations for the perfect diatomic 2D structure from Eq. (2.1) may be cast in the matrix form as

$$
\left(\Omega^{2} I-D\left(z, \varphi_{y}\right)\right)|\boldsymbol{U}\rangle=|0\rangle .
$$

$\Omega$ is the energy of the vibration modes of the perfect diatomic 2D lattice. It contains the characteristic frequency of the perfect lattice, which is defined by $k$ and $m$. Here $\Omega$ is expressed by $\Omega^{2}=\omega^{2} / \omega_{0}^{2}$, and $\omega_{0}^{2}=\frac{k(A, A)}{m_{A}}$. $D\left(z, \varphi_{y}\right)$ is the dynamical matrix characteristic of the system, and $I$ is its corresponding unit matrix. For the configuration under study the matrices are reduced to the size $(8 \times 8)$.

The $D$ elements are expressed as a function of the $z=$ $\exp \left(\mathrm{i} \boldsymbol{q}_{x} \boldsymbol{a}\right)$ and $\varphi_{y}=\exp \left(\mathrm{i} \boldsymbol{q}_{y} \boldsymbol{a}\right)$, whereby $\left(\boldsymbol{q}_{x}, \boldsymbol{q}_{y}\right)$ are the reciprocal lattice wave vectors, respectively in the $x$ and $y$ directions, and $a$ is the lattice parameter.
By diagonalizing Eq. (2.2), for $|z|=1$, we obtain the eight (8) vibration modes of the diatomic structure presented in Fig. 1.

Among the dispersion curves, we note only two (2) acoustical modes. The others are optical ones. They are ranged from bottom to top in a numerical order. These modes are presented as a function of the normalized wave vector $q_{x}$ that runs over high symmetry points (HSP) in the first Brillouin zone as depicted in Fig. 2. All the modes are symmetric regarding to the Brillouin zone center.

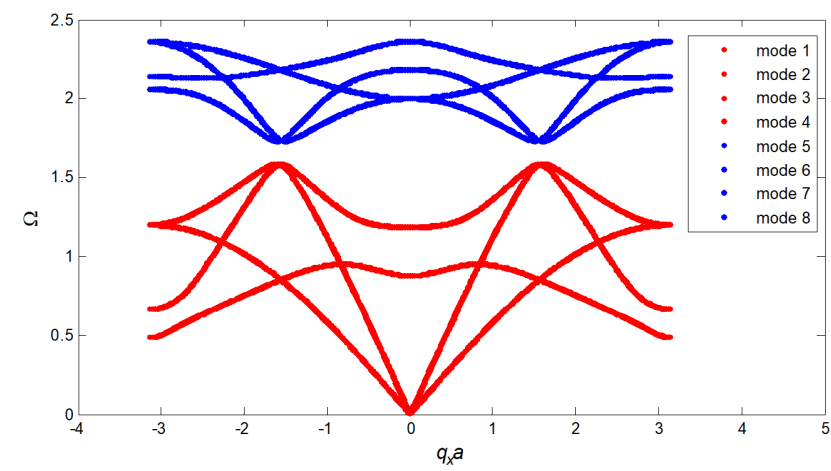

Fig. 2. Phonons dispersion modes for the 2D diatomic lattice depicted in Fig. 1.

Each vibration mode propagates in its specific interval frequency. We notice the existence of zones where some modes are recovered. These zones signify that the above modes can be excited simultaneously.

The dispersion phonon modes are strongly dependant on the variation in atomic masses and the force elastic interactions between the sites that form the unit cell.

In addition, the unit cell, of the diatomic system given in Fig. 1, is composed by four (4) atoms, two $A$ and two $\ell^{\prime}$ sites, the dispersion curves corresponding to the $\ell$ sites are the modes numerated from 1 to 4 , in the bottom modes. The other modes, on the top, from 5 to 8 , correspond to the $B$ vibration modes. The phonons modes of the two different types of atoms are separated by forbidden band.

In addition, the group velocities can be determined, directly, by calculating the slopes of the dispersion curves. When the slopes are negatives, these signify that the elastic waves changes the sense of propagation.

\section{Neural network model}

An artificial neural network is a model that imitates the mathematical behaviours of biological nervous systems. Definitely, in the neural approach, the formal neuron is the basic processing elements of neural networks. In a roughly similar way, an artificial neural network, based on a simplified model of the neuron, is thus composed of a very large number of small and similar processing units called artificial neurons "formal neuron". These are interconnected by weighted links, i.e., connections, also called "synapses" according to the corresponding biological term. The nonlinear characteristic displayed by 
neurons is represented by a differentiable function called transfer functions. Mathematically, the output of the $i$ th neuron in the $k$-th layer of a network is [18]:

$$
x_{i}^{(k)}=f^{(k)}\left(\sum_{j=1}^{\tau} w_{i j}^{(k)} x_{j}^{(k-1)}\right) .
$$

Here $x_{i}^{(k)}$ refers to the network response at the $k$-th layer, $w_{i j}$ are connection links between the input layer and the hidden layer, and $\tau$ is the number of neurons in the layer $(k-1)$. The node of each neuron in the network is associated with a continuously differentiable transfer function given by $f^{(k)}$.

\subsection{Database collection}

In this subsection, we describe the neural approach used for predicting the 2D structure of crystal surfaces. The training and evaluation of neurocomputing model (NCM) involve three phases that include: first, collecting the database, which characterize the input-output unit of the tasks under study. Second, constructing the proposed network topology for the given data. Third, the performance of the proposed neural architecture is checked. The essentials of these steps are discussed in the next section.

Most of the common material crystallizations are body-centered cubic (bcc), face-centred cubic (fcc), hexagonal close packed (hcp), etc. In these structures, there are in principle multitude possible positions on their surfaces. Moreover, it is achievable to determine the atomic arrangement on, a particular, crystal surfaces. The latter depends on how the bulk structure is intersected.

To collect the data set for NCM, let us consider some simple examples illustrating the surface structures. For illustrations, Fig. 3 and Fig. 4 show several 2D structures defining some surfaces having hexagonal and square arrangements, respectively. The $2 \mathrm{D}$ lattice of these surfaces shows gray circles which map the substrate atoms and blue circles which map the adsorbate atoms.

As conclusion, there are different structures which represent different possible arrangements that can be exposed on the surface. The solutions of the dynamic equations (an eigenvalue problem) for different surface arrangements give rise to many physical parameters that will be used to generate the NCM. The descriptions of data collection are discussed in the next subsection.

\subsection{Description of the collected data}

1. The first input unit is organized into ten (10) blocks: (i) the first eight (8) blocks contain the values of angular frequency vectors $\Omega_{1}\left(z, \varphi_{y}\right), \Omega_{2}\left(z, \varphi_{y}\right), \Omega_{3}\left(z, \varphi_{y}\right), \Omega_{4}\left(z, \varphi_{y}\right)$, $\Omega_{5}\left(z, \varphi_{y}\right), \Omega_{6}\left(z, \varphi_{y}\right) \Omega_{7}\left(z, \varphi_{y}\right)$ and $\Omega_{8}\left(z, \varphi_{y}\right)$ generated from the dynamic equation. Each of frequency modes is organized into six (6) neurons that is used as training data. (ii) The ninth (9) block contains the values of wave vector $z=\varphi_{x}$ over HSP in the first Brillouin zone. The values are organized

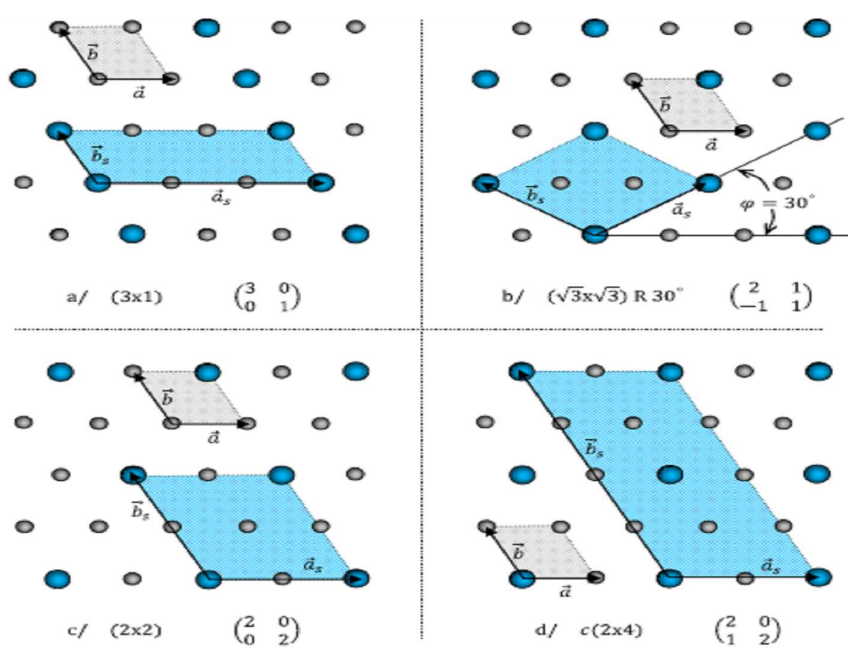

Fig. 3. The Wood and matrix notation for some superlattices on a hexagonal $2 \mathrm{D}$ lattice.

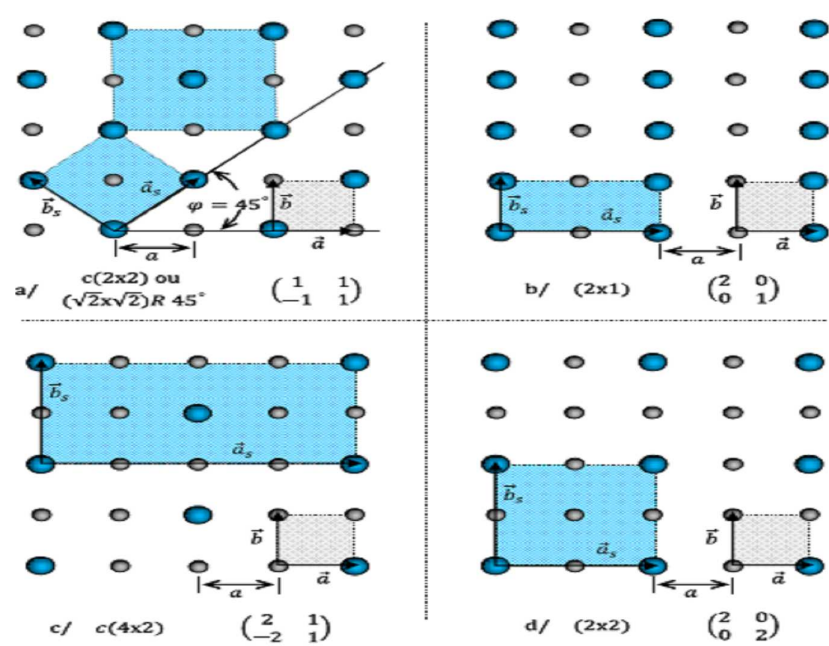

Fig. 4. The Wood and matrix notation for some superlattices on a square $2 \mathrm{D}$ lattice.

into six (6) neurons. (iii) The last block contains the velocity data of each frequency response. Since the velocity depends on the frequency modes, the neuron number in this block will be the number of modes times the neuron units used for each mode. In total, we get 48 neurons for the velocity block.

2. The neuron number in each block represents the training units. We sample the wave vector to yield 240 training data generated uniformly over six (6) units which refers to the neuron number in each block.

3. We have taken the lattice spacing data in a separated unit (2), since for each value of a given lattice spacing [12] we have to resolve the eigenvalue problem in Eq. (2.1). 
4. The output data have been classified according to the matrix and Wood notations. For each structure, we select the output data as a vector whose elements are extracted from the matrix and Wood notations. Moreover, whilst dealing with the output data, the HSP's path should be in accord with matrix and wood's notations since each notation has its related HSP path. For these reasons, the training output vector is given as $\left[a, b, c, d, \frac{\theta}{10}, \gamma, \delta, \rho, \sigma\right]$, where the 4 elements $(a, b, c$, and $d$ ) represent the matrix notation, $\theta$ is the angle of rotation, which aligns the unit cell of the reconstructed surface (the Wood notation). Furthermore, $\gamma, \delta, \rho$ and $\sigma$ refer to HSP path. As an example, the corresponding vector of substrate unit $c(3 \times 1): \sqrt{2} \times \sqrt{2} R 45$ is a vector whose elements are $[3,0,0,1,4.5, \gamma, \delta, \rho, \sigma]$.

A schematic representation of the network topology is shown in Fig. 5.

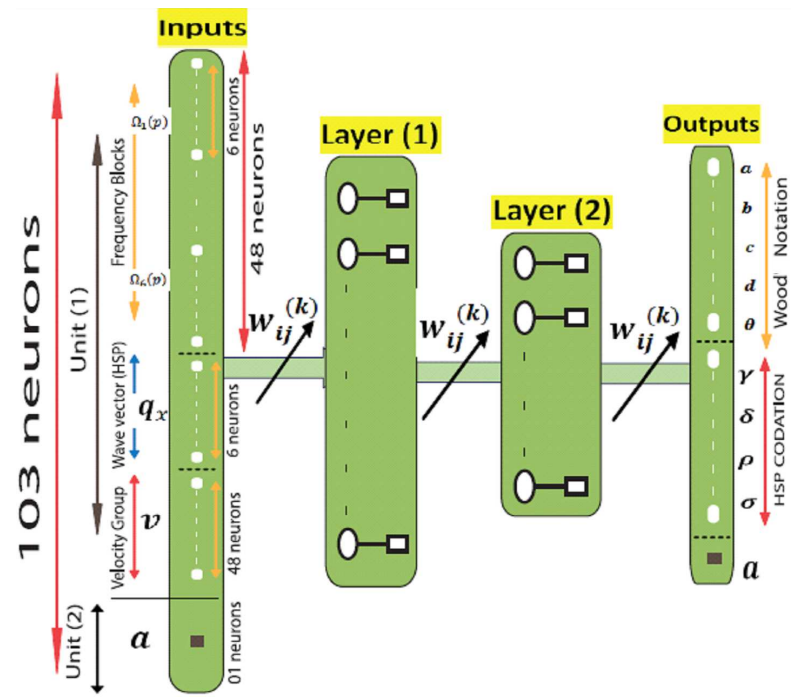

Fig. 5. A schematic representation of the network topology used in this approach, the model is given by three hidden layers connected in a feed forward manner.

As the inputs-outputs set has been coded as explained above, the neurocomputing should present 103 neurons in the input layer (forty-eight 48 neurons for frequency modes, forty-eight (48) neurons for group velocity, six (6) neurons for wave vector, and one (1) neuron for lattice spacing). The hidden layer is powered with 205 neurons, while the output layer contains 10 neurons. The initialization of the network weights and bias has been generated by using Nguyen and Widrow's initialization algorithm [19]. In this study, the Mexican hat wavelet function, given as $f(u)=\left(1-u^{2}\right) \mathrm{e}^{-\left(u^{2} / 2\right)}$, has been employed at hidden layers. However, for the output layer a linear function has been used as activation, i.e., $f(u)=u$. Additionally, in this contribution, the Levenberg-Marquardt algorithm has been used to train the neural network connection links. The training and optimization of neural networks to perform the training process is well documented in the literatures [20, 21].

\section{Results}

In this section, we illustrate the performance of our model. The letter is done by testing the trained network to evaluate its ability in predicting the $2 \mathrm{D}$ lattice structures. For a better validation of our programs, the performance of NCM is checked by some classification of absorption sites on fcc (111), hcp (0001), ccp (111), ccp (110) and bbc (110). For more details, see Refs. [22-24].

We can select from theses absorptions a bunch of possible arrangements [25-29] that will be used as testing samples.

- $\mathrm{V} / \mathrm{Pt}(111)$ given by the substrate unit $c(2 \times 1)$ : $\sqrt{3} \times \sqrt{3} R 30$. During the training stage, the output unit, in the network language of such a substrate, was coded by a training vector whose elements were $[2,0,0,1,3.00]$. We have shown only the first 5 elements of the output data in order to be able to compare the network response with the matrix and Wood notations.

- $\mathrm{Au} / \mathrm{Pd}(100)$ given by the class $c(2 \times 2): \sqrt{2} \times$ $\sqrt{2} R 45$. During the training stage, the output of such a class was coded by the training elements $[2,0,0,2,4.50]$.

- $\mathrm{Sm} / \mathrm{Rh}(100)$ given by the unit $c(2 \times 2): 5 \sqrt{2} \times$ $\sqrt{2} R 45$. The training elements of such a unit were given by the training elements $[2,0,0,2,4.50]$.

- Ce/Pt (111) given by $c(2 \times 2): \sqrt{3} \times \sqrt{3} R 45$. The training elements of this kind of class were chosen to be as $[2,0,0,2,4.50]$.

- $\mathrm{Ce} / \mathrm{Rh}(110)$ given by $c(2 \times 1): \sqrt{3} \times \sqrt{3} R 30$. The training elements of this kind of unit were $[2,0,0,1,3.00]$.

- $\mathrm{Sb} / \mathrm{Ag}(110)$ given by the class $c(2 \times 2): 2 \sqrt{3} \times$ $2 \sqrt{3} R 30$. The training elements corresponding of this class were given as $[2,0,0,2,3.00]$.

For simulation stage, the given structures are being used as testing steps. This stage allows us to check the reliability of the proposed NCM in classifying these arrangements according to the Wood and matrix notations.

First, we keep the same architecture of the trained neural network. Next, we prepare the input units of the given testing samples. The testing dataset contains the required data about frequency modes, wave vectors, group velocity, and lattice spacing. Finally, the simulation results, which provided the output vectors through NCM, are resumed in Table I, given below. 
TABLE I

The matrix and Wood notations compared to the first five elements of network responses according to each testing arrangement of NCA output vectors related to the testing data.

\begin{tabular}{c|l|l}
\hline \hline & \multicolumn{1}{|c}{$\mathrm{M} \mathrm{\&} \mathrm{W}$} & \multicolumn{1}{c}{ NCA } \\
\hline $\mathrm{V} / \mathrm{Pt},(111)$ & {$[2,0,0,1,3.00$} & {$[2.81,0.12,-0.24,0.91,2.63]$} \\
$\mathrm{Au} / \mathrm{Pd},(100)$ & {$[2,0,0,2,4.50]$} & {$[2.37,-0.08,0.39,2.48,3.92]$} \\
$\mathrm{Sm} / \mathrm{Rh},(100)$ & {$[2,0,0,2,4.50]$} & {$[1.78,-0,47,-0.26,1.76,5.08]$} \\
$\mathrm{Ce} / \mathrm{Pt},(111)$ & {$[2,0,0,2,4.50]$} & {$[1.83,0.32,-0.47,1.79,4.15]$} \\
$\mathrm{Ce} / \mathrm{Rh},(110)$ & {$[2,0,0,1,3.00]$} & {$[2.76,0.08,0.41,1.27,3.12]$} \\
$\mathrm{Sb} / \mathrm{Ag}, 110)$ & {$[2,0,0,2,3.00]$} & {$[2.19,-0.23,-0.18,2.46,3.72]$} \\
$\mathrm{V} / \mathrm{Pt},(111)$ & {$[2,0,0,1,3.00$} & {$[2.22,-0.29,0.34,1.16,3.86]$}
\end{tabular}

According to the output vectors related to the testing data, given in Table I, we quote the following remarks.

- The NCM estimation related to the testing data of $\mathrm{V} / \mathrm{Pt}(111)$ is $[2.81,0.12,-0.24,0.91,2.63]$. The latter is close to the vector $[2,0,0,1,3.00]$. Hence, the network indicates that the structure is classified as $C(2 \times 1): \sqrt{3} \times \sqrt{3} R 30$.

- The NCM output related to the testing data of $\mathrm{Au} / \mathrm{Pd}(111)$ is $[2.37,0.08,0.39,2.48,3.92]$. The latter is close to the vector $[2,0,0,2,4.50]$. Hence, the network indicates that the structure is classified as $C(2 \times 2): \sqrt{2} \times \sqrt{2} R 45$.

- The NCM's estimation related to the testing data of $\mathrm{Ce} / \mathrm{Rh}(110)$ is $[2.76,0.08,0.41,1.27,3.12]$. The latter is close to the vector $[2,0,0,1,3.50]$. Hence, the network indicates that the structure is classified as $C(2 \times 2): \sqrt{3} \times \sqrt{3} R 30$.

Additionally, the lattice spacing calculations given by the NCM are compared to the values of testing crystals: $\mathrm{Pt}$, $\mathrm{Pd}, \mathrm{Au}, \mathrm{Ce}, \mathrm{Rh}$, and $\mathrm{Cb}$. In Table II, we have summarized the network responses for the elements under test.

\section{TABLE II}

The NCM calculation of lattice spacing versus its real cell value [12]; [pm].

\begin{tabular}{c|c|c|c|c|c|c}
\hline \hline & $\mathrm{Pt}$ & $\mathrm{Pd}$ & $\mathrm{Au}$ & $\mathrm{Ce}$ & $\mathrm{Rh}$ & $\mathrm{Sb}$ \\
\hline network & 302.015 & 405.251 & 425.529 & 381.49 & 213.051 & 185.34 \\
cell & 392.42 & 389.07 & 407.82 & 362 & 250.71 & 206
\end{tabular}

From Table I, the results illustrate that the neural network has well recognized and classified possible arrangements of testing samples. This study confirmed that using the Wood method along with matrix notations is a very useful approach to distinguish the different arrangements given in training stage as well as simulation stage. However, the model was not accurate to determine the lattice spacing of the same testing samples. In fact, for the first illustration given in Table I, the NCM was more accurate because the connection links are quite important as shown in Fig. 5. It means that the neural model contains raising neurons in the output-layer unit. The latter permitted us to have more connections in terms of weights; consequently, the NCM were more reliable in predicting the arrangements of testing samples. On the other hand, the connection links assigned to the lattice spacing is only one neuron in the output unit as Fig. 5 depicts. For this reason, the simulation stage was hopeless in evaluating the lattice spacing.

The results can be improved by acting on several parameters: (i) the training data quality, for the lattice spacing, need to be coded in a very useful way. For example, each lattice data have to be broadened by giving each crystal a specific vector that refers to its lattice spacing values. (ii) We have to increase the neuron number in the hidden layer to get the optimal neural network model that would be adequate to ensure a higher accuracy.

\section{Summary and conclusion}

A theoretical study to predict the possible arrangements of crystal surfaces were reported. The model was based on dynamic vibration and the Wood notation. These latter allowed collecting enough data to feed the input-output units of neural network. This contribution has shown the capability of the NCM to recognize and classify the possible arrangements of crystal surfaces.

Neural approaches involve collecting the input-output set in the training stage. The matrix and Wood notations have given a helpful way to classify the arrangements on surfaces for the testing crystals. Additionally, the network response was in a good agreement with the matrix and Wood notations. The network connection links were initialized by Nguyen and Widrow's method, and the training steps were accomplished by using the Levenberg-Marquardt and back-propagation algorithms.

To sum up, the model would be very helpful while dealing with experimental data. In fact, it would be able to predict the surface structures of the given data. We have to feed the given experimental data and let the NCM computes the output units. The latter leads to extract the classes of each data and then compare them with the matrix and Wood notations.

As a conclusion, inspired by the operation of nervous systems, the artificial neural network currently constitutes one of the most efficient tools to process alternative and increasingly competent application in many fields.

\section{References}

[1] S.Y. Kung, J.S. Taur, IEEE Trans. Neural Network 6, 170 (1995).

[2] H.K. Lam, U. Ekong, H. Liu, B. Xiao, H. Araujo, S.H. Ling, K.Y. Chan, Neurocomputing 144, 367 (2014).

[3] A. Nazemi, M. Dehghan, Neurocomputing 152, 369 (2015).

[4] A.V. Savchenko, Neural Networks 46, 227 (2013). 
[5] M.A.Z. Raja, U. Farooq, N.I. Chaudhary, A.M. Wazwaz, Appl. Soft Comput. 38, 561 (2016).

[6] M.A.Z. Raja, R. Samar, Appl. Math. Model. 40, 5964 (2016).

[7] J.A. Khan, M.A.Z. Raja, M.M. Rashidi, M.I. Syam, A.M. Wazwaz, Connect. Sci. 27, 377 (2015).

[8] M.A.Z. Raja, Connect. Sci. 26, 195 (2014).

[9] M.A.Z. Raja, R. Samar, T. Haroon, S.M. Shah, Appl. Math. Mech. 36, 1611 (2015).

[10] M.A.Z. Raja, R. Samar, Neurocomputing 124, 178 (2014).

[11] M.A.Z. Raja, J.A. Khan,T. Haroon, J. Taiwan Inst. Chem. Eng. 48, 26 (2015).

[12] C. Kittel, Introduction to Solid State Physics, John Wiley \& Sons, 2008.

[13] G. Belkacemi, B. Bourahla, Superlatt. Microstruct. 85, 226 (2015).

[14] A. Khater, B. Bourahla, R. Tigrine, J. Phys. 92, 012032 (2007).

[15] M.A. Ghantous, A. Khater, J. Euro. Phys. B 12, 335 (1999).

[16] C. Berthod, F. Gagel, K. Maschke, J. Phys. Rev. B 50, 18299 (1994).

[17] F. Gagel, K. Maschke, Phys. Rev. B 52, 2013 (1995).
[18] F.M. Hum, I. Kostanic, Principles of Neurocomputing for Science and Engineering, McGraw Hill, New York 2001.

[19] D. Nguyen, B. Widrow, Neural Networks 3, 21 (1990).

[20] B.M. Wilamowski, H. Yu, IEEE Trans. Neural Network 21, 930 (2010).

[21] P. Chandra, Y. Singh, Neurocomputing 61, 429 (2004).

[22] I. Richard, M. Hardcover, Principles of Adsorption and Reaction on Solid Surfaces, John Wiley \& Sons, 1996.

[23] S. Schwegmann, W. Tappe, U. Korte, Surf. Sci. 334, 55 (1995).

[24] H. Over, Prog. Surf. Sci. 58, 249 (1998).

[25] J. Breitbach, D. Franke, G. Hamm, C. Becker, K. Wandelt, Surf. Sci. 507, 18 (2002).

[26] M. Juel, B.T. Samuelsen, M. Kildemo, S. Raaen, Surf. Sci. 601, 2917 (2007).

[27] R. Chadli, A. Khater, R. Tigrine, J. Appl. Phys. 57 21303 (2012).

[28] B. Vermang, M. Juel, S. Raaen, J. Phys. Rev. B 73, 033407 (2006).

[29] C. Félix, G. Vandoni, W. Harbich, J. Buttet, R. Monot, J. Phys. Rev. B 54, 23 (1996). 